\title{
Macroscopic quantum fluctuations in noise-sustained optical patterns
}

\author{
Roberta Zambrini, ${ }^{1}$ Stephen M. Barnett, ${ }^{2}$ Pere Colet, ${ }^{1}$ and Maxi San Miguel ${ }^{1}$ \\ ${ }^{1}$ Instituto Mediterráneo de Estudios Avanzados, IMEDEA (CSIC-UIB), Campus Universitat Illes Balears, \\ E-07071 Palma de Mallorca, Spain \\ ${ }^{2}$ Department of Physics and Applied Physics, University of Strathclyde, 107 Rottenrow, Glasgow G4 ONG, Scotland
}

(Received 6 August 2001; revised manuscript received 23 October 2001; published 15 January 2002)

\begin{abstract}
We investigate quantum effects in pattern formation for a degenerate optical parametric oscillator with walk-off. This device has a convective regime in which macroscopic patterns are both initiated and sustained by quantum noise. Familiar methods based on linearization about a pseudoclassical field fail in this regime and new approaches are required. We employ a method in which the pump field is treated as a $c$-number variable but is driven by the $c$-number representation of the quantum subharmonic signal field. This allows us to include the effects of the fluctuations in the signal on the pump, which in turn act back on the signal. We find that the nonclassical effects, in the form of squeezing, survive just above the threshold of the convective regime. Further, above threshold, the macroscopic quantum noise suppresses these effects.
\end{abstract}

DOI: 10.1103/PhysRevA.65.023813

\section{INTRODUCTION}

Nonlinear optics has provided an ideal testing ground for ideas in both nonlinear dynamics and quantum optics. It provides fast nonlinearities and a degree of control that allow fundamental dynamical systems to be realized and nonlinear phenomena, such as pattern formation to be demonstrated [1]. It also provides systems with very low levels of noise so that fluctuations can be limited by quantum effects. The combination of these features has led to the study of quantum phenomena in optical pattern formation $[2,3]$ and of noisy precursors of the patterns, which have been termed quantum images [4-6]. The accurate modeling of such quantum nonlinear systems presents a significant challenge. Pattern formation and dynamics are usually associated with excitation of a large number of transverse modes and a fully quantum description of each of these is required in order to properly treat the quantum fluctuations. The Heisenberg picture produces a hierarchy of coupled nonlinear operator equations that usually defies analysis. The preferred method to date has been to linearize the quantum fluctuations about a classical field amplitude that usually takes a constant value below threshold [6,7], but may be associated with a stable pattern above threshold [8].

A more difficult situation arises if the system displays macroscopic features driven by noise. In such cases we cannot expect linearization of the quantum fluctuations to give reliable results and a new approach is needed. A simple device demonstrating macroscopic, noise-driven patterns is the degenerate optical parametric oscillator in the presence of walk-off. The semiclassical analysis of this device reveals a region of convective instability, above the threshold for oscillation, in which noise sustained structures are seen in the transverse field distribution $[9,10]$. The aim of this paper is to develop a suitable approximation scheme with which to model quantum effects in the parametric oscillator in this regime of operation.

The convectively unstable regime is characterized by an amplification and flow of fluctuations [11]. In systems in which the spatial reflection invariance is broken by the presence of a group-velocity term, local perturbations of the
PACS number(s): 42.50.Lc, 42.50.Dv, 42.50.Ct, 42.65.Sf

steady state can be advected more rapidly than their growth rate. If the system is deterministic then at any fixed point any initial localized perturbation decays and the system approaches the undisturbed steady state. In this case macroscopic patterns can arise and be observed only if noise is continuously applied, the structure now being regenerated at any time, hence the name noise-sustained patterns. These structures are the result of noise self-organization, with magnification factors of several orders of magnitude. They are thus interesting candidates for the study of quantum correlations in spatially structured systems.

Any system with an advection (or drift or walk-off) term that is also not translationally invariant will, in general, be convectively unstable when operating sufficiently close to and above the onset of the instability of the steady state. Hence, this type of instability has been predicted in a number of optical systems including Kerr media with a tilted pump $[9,12]$ and optical parametric oscillators $(\mathrm{OPO})$ with walk-off [12-14].

Modeling quantum effects in the regime of convective instability for a nonlinear optical device presents a double challenge. First, the system has a broad spectrum both in the frequency (at a fixed point) and in wave vectors (far field at a fixed time), thus it cannot be studied within a few-mode approximation. Second, we should be able to follow the evolution of the fluctuations from the microscopic level through the amplification into the macroscopic pattern. In order to do this we introduce, in Sec. IV, a suitable nonlinear approximation with which to treat the convective regime of a degenerate optical parametric oscillator. In order to fix the terms of reference for this approximation we begin, in Sec. II, with a review of the semiclassical features of the device and its convective instability. This is followed, in Sec. III, by a quantum description of the device. Once we have introduced our method we discuss quantum features of the device in its various regimes of operation (Sec. V), paying particular attention to the demanding convective regime (Sec. VI).

\section{SEMICLASSICAL DESCRIPTION AND CONVECTIVE REGIME}

We consider a degenerate optical parametric oscillator (DOPO), a device consisting of a cavity filled with a $\chi^{(2)}$ 
nonlinear medium, which converts a pump at frequency $2 \omega$ into a subharmonic signal at frequency $\omega$. The possibility of phase matching the down-conversion process depends on the birefringence of the crystal that provides a difference of the refractive index for differently polarized fields. We can exploit this difference in order to avoid the effects of dispersion by selecting the same index of the refraction for the pump and signal $n_{2 \omega}=n_{\omega}$. In this paper we consider type-I phase matching for which ordinary polarized pump photons are down-converted to produce pairs of extraordinary polarized photons that are degenerate both in the frequency and in polarization.

In anisotropic media rays do not necessarily travel in a direction perpendicular to their wavefronts [15]. As a consequence the extraordinarily polarized signal generated in our DOPO will walk off, that is it will propagate in the transverse direction relative to the ordinarily polarized pump. This transverse walk-off effect is described in the dynamical equations by a term that accounts for a velocity relative to the frame of reference fixed by a pump of finite transverse width.

The quantum effects we wish to study are associated with the convective regime and it is important to define this carefully. The different regimes of operation of a DOPO can be understood within a semiclassical theory and this section provides a brief (semiclassical) analysis of the convective and other regimes. A more complete discussion can be found in Ref. [13]. The intracavity field is described by two slowly varying complex field amplitudes $A_{0}(\vec{x}, t)$ and $A_{1}(\vec{x}, t)$ for the pump and the signal, respectively. These depend on the transverse spatial coordinates $\vec{x}=(x, y)$ and the time $t$. Within the paraxial approximation (for propagation in the $z$ direction), the mean-field limit and for single longitudinalmode operation the dynamical equations become $[13,16,17]$

$$
\begin{aligned}
\partial_{t} A_{0}(\vec{x}, t)=- & \gamma_{0}\left[1+i \Delta_{0}-i a_{0} \nabla^{2}\right] A_{0}(\vec{x}, t)-\frac{g}{2} A_{1}^{2}(\vec{x}, t) \\
+ & E_{0}(\vec{x})+\epsilon_{0} \xi_{0}(\vec{x}, t) \\
\partial_{t} A_{1}(\vec{x}, t)=- & \gamma_{1}\left[1+i \Delta_{1}-i a_{1} \nabla^{2}-v \partial_{y}\right] A_{1}(\vec{x}, t) \\
& +g A_{0}(\vec{x}, t) A_{1}^{*}(\vec{x}, t)+\epsilon_{1} \xi_{1}(\vec{x}, t)
\end{aligned}
$$

Here $\xi_{i}(i=0,1)$ are additive Gaussian white sources of noise, with nonvanishing correlations of the form

$$
\left\langle\xi_{i}(\vec{x}, t) \xi_{j}^{*}\left(\vec{x}^{\prime}, t^{\prime}\right)\right\rangle=\delta_{i j} \delta\left(\vec{x}-\vec{x}^{\prime}\right) \delta\left(t-t^{\prime}\right) .
$$

The level of noise introduced is fixed by the parameters $\epsilon_{0}$ and $\epsilon_{1}$. Our fully quantum analysis will produce equations of similar form in which these parameters are fixed. $E_{0}$ is the amplitude of the driving field, which we take to be real. The remaining parameters in these equations are the cavity decay rates $\gamma_{i}$, the cavity detunings $\Delta_{i}$, the diffraction $a_{i}$, the walk-off $v$, and the nonlinear coefficient $g$. It is convenient to introduce scaled variables

$$
t^{\prime}=\gamma t, \quad \vec{x}^{\prime}=\frac{\vec{x}}{\sqrt{a}}, \quad v^{\prime}=\frac{v}{\sqrt{a}},
$$

$$
A_{i}^{\prime}=\frac{g}{\gamma} A_{i}, \quad E_{0}^{\prime}=\frac{g}{\gamma^{2}} E_{0}, \quad \epsilon_{i}^{\prime}=\frac{g}{\gamma^{3 / 2} a^{D / 4}} \epsilon_{i},
$$

where we have restricted the cavity decay rates and diffraction coefficients such that $\gamma=\gamma_{0}=\gamma_{1}$ and $a=a_{0}=a_{1} / 2$. Our equations are valid either for one or two transverse spatial dimensions $(D=1,2)$. On omitting the primes, our amplitude Eqs. (1) and (2) become

$$
\begin{aligned}
\partial_{t} A_{0}(\vec{x}, t)=- & {\left[1+i \Delta_{0}-i \nabla^{2}\right] A_{0}(\vec{x}, t)-\frac{1}{2} A_{1}^{2}(\vec{x}, t)+E_{0}(\vec{x}) } \\
& +\epsilon_{0} \xi_{0}(\vec{x}, t) \\
\partial_{t} A_{1}(\vec{x}, t)= & -\left[1+i \Delta_{1}-2 i \nabla^{2}-v \partial_{y}\right] A_{1}(\vec{x}, t) \\
& +A_{0}(\vec{x}, t) A_{1}^{*}(\vec{x}, t)+\epsilon_{1} \xi_{1}(\vec{x}, t)
\end{aligned}
$$

For a uniform driving field $E_{0}$, Eqs. (5) and (6) admit the homogeneous stationary solution

$$
A_{0}^{s}=\frac{E_{0}}{1+i \Delta_{0}}, \quad A_{1}^{s}=0 .
$$

The threshold for parametric oscillation can be determined by a linear stability analysis of this solution. The linearized equations for the signal and pump fluctuations $\delta A_{1}(\vec{x}, t)$ $=A_{i}(\vec{x}, t)-A_{i}^{s}(i=0,1)$ are decoupled, and the fluctuations of the pump are always damped. For the signal, we consider perturbations of the form $\exp [\vec{k} \cdot \vec{x}+\lambda(\vec{k}) t]$ and find the dispersion relation

$$
\lambda_{ \pm}(\vec{k})=-1+i v k_{y} \pm \sqrt{F^{2}-\left(\Delta_{1}+2|\vec{k}|^{2}\right)^{2}},
$$

where we have introduced a scaled pump

$$
F=\frac{E_{0}}{\sqrt{1+\Delta_{0}^{2}}}
$$

We find that there is an instability at $F=1$. For $F<1$, $\operatorname{Re}(\lambda)<0$ and the solution (7) is absolutely stable. For $F$ $>1$, there is a positive growth rate of fluctuations $\left[\operatorname{Re}\left(\lambda_{+}\right)\right.$ $>0]$ that takes a maximum value for $\left|\vec{k}_{c}\right|=\sqrt{-\Delta_{1} / 2}$ if the signal detuning is negative $\left(\Delta_{1}<0\right)$, and for $k=0$ if $\Delta_{1}$ $>0$. In this paper we are interested in the case of pattern formation and we restrict our analysis to the case $\Delta_{1}<0$. The instability at $F=1$ when $v=0$ is a Turing instability, in which a stationary pattern appears [16]. If $v \neq 0$ then the eigenvalue becomes complex and we find a Hopf bifurcation in which a traveling pattern emerges [13].

The direction of instability is determined by the eigenfunctions $V_{ \pm}(\vec{k},-\vec{k})$ of the linear problem $\partial_{t} V_{ \pm}(\vec{k},-\vec{k})$ $=\lambda_{ \pm}(\vec{k}) V_{ \pm}(\vec{k},-\vec{k})$. Solving this gives

$$
\begin{gathered}
V_{ \pm}(\vec{k},-\vec{k})=e^{i \Phi_{ \pm} \delta A_{1}(\vec{k}) \pm \delta A_{1}^{*}(-\vec{k}),} \\
e^{i \Phi_{ \pm}(\vec{k})}= \pm \frac{i \Delta_{1}+2 i|\vec{k}|^{2} \mp \sqrt{\left|A_{0}^{s}\right|^{2}-\left(\Delta_{1}+2|\vec{k}|^{2}\right)^{2}}}{A_{0}^{s}} .
\end{gathered}
$$




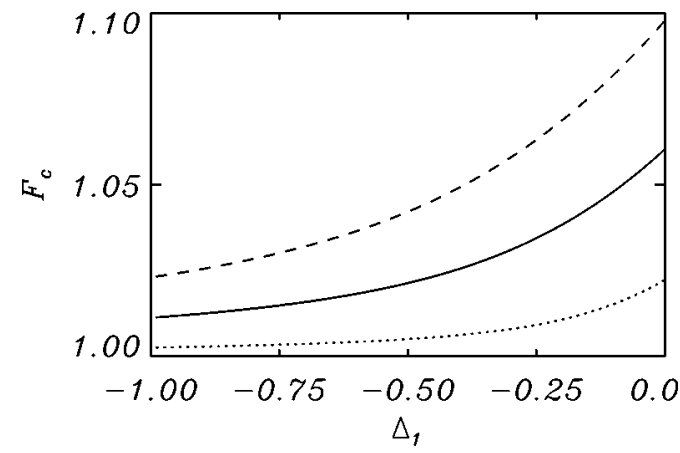

FIG. 1. Stability diagram as a function of the signal detuning $\Delta_{1}$. The different lines correspond to the threshold of absolute instability $F_{c}$ for different values of the walk-off parameter: $v=0.2$ (dotted line), $v=0.42$ (continuous line), and $v=0.6$ (dashed line). When $F<1$ the solution (7) is absolutely stable, while for $1<F$ $<F_{c}$ the solution is convectively unstable.

The solution $V_{+}(\vec{k},-\vec{k})$ gives the direction of amplification of fluctuations, while fluctuations are damped for $V_{-}(\vec{k}$, $-\vec{k})$. In particular, for the critical wave vector $\left|\vec{k}_{c}\right|$ and for a real pump $E_{0}$ and $\Delta_{0}=0$, we obtain $V_{ \pm}\left(\vec{k}_{c},-\vec{k}_{c}\right)$ $=\delta A_{1}\left(\vec{k}_{c}\right) \pm \delta A_{1}^{*}\left(-\vec{k}_{c}\right)$. Therefore, in this case, the difference of real parts and the sum of imaginary parts of the field in $\vec{k}_{c}$ and $-\vec{k}_{c}$ will show damped fluctuations at the threshold. We also note that the instability direction is independent of the walk-off term.

Above the instability threshold $(F=1)$ the steady state (7) is convectively unstable: any perturbation grows while traveling in the direction fixed by the walk-off term and eventually leaves the system [13]. In this regime a continuous perturbation, such as a source of noise, gives rise to a noisesustained pattern consisting in disordered traveling stripes in the signal. On increasing the pump a second threshold is reached at $F=F_{c}$. Beyond this threshold the pattern is sustained by the nonlinear dynamics, being also present in the absence of perturbations, once it is formed. The state (7) is absolutely unstable in this regime [13]. In Fig. 1 we plot the result of the calculation of the absolute instability threshold as a function of the signal detuning $\Delta_{1}$, for different values of the walk-off parameter $v$.

Walk-off has three main effects in this process of pattern formation $[12,13]$. The first is the existence of the convective regime in which patterns are sustained by the noise. Second is that it breaks the rotational symmetry, favoring the formation of stripes orthogonal to the walk-off direction and traveling in this direction. Third, the selected wave vector, that is the most intense mode $\vec{k}_{M}$ of the pattern, depends on the walk-off parameter. An approximate expression for $\vec{k}_{M}$ can be obtained in the context of front propagation into an unstable state $[10,18]$.

There are two important characteristics of the noise sustained patterns that exist in the convective regime. The first is a broad spectrum, both in the frequency and in wave vectors [19]. Second is the presence of macroscopic amplified signal fluctuations around the unstable reference state (7). These characteristics imply that the convective regime can- not be studied within a few-mode approximation, because many modes contribute significantly to the spectral properties. The presence of macroscopic fluctuations also invalidates approximations based on linearization schemes. These facts make a quantum formulation of the convective regime especially difficult. We face a situation in which nonlinearities determine the dynamics of fluctuations around the reference state, with fundamental quantum noise being amplified by several orders of magnitude to produce a macroscopic pattern in the signal.

\section{QUANTUM FORMULATION}

In the quantum formulation of the DOPO the intracavity pump and signal fields are given by operators $\hat{A}_{0}(\vec{x}, t)$ and $\hat{A}_{1}(\vec{x}, t)$ that satisfy standard equal-time commutation relations [6]

$$
\left[\hat{A}_{i}(\vec{x}, t), \hat{A}_{j}^{\dagger}\left(\vec{x}^{\prime}, t\right)\right]=\delta_{i j} \delta\left(\vec{x}-\vec{x}^{\prime}\right),
$$

where the indices $i, j$ stand for 0,1 . Following the techniques described in [6], we can introduce a model Hamiltonian for the device. This will include the effects of diffraction together with the driving by a real, classical external field, nonlinear interaction between the fields and cavity damping. Our model, however, also requires that we take account of the effects of the walk-off. The resulting Hamiltonian gives, on making the usual Markov approximation, the coupled Heisenberg equations

$$
\begin{gathered}
\partial_{t} \hat{A}_{0}(\vec{x}, t)=-\left[\gamma_{0}\left(1+i \Delta_{0}\right)-i a_{0} \nabla^{2}\right] \hat{A}_{0}(\vec{x}, t) \\
-\frac{g}{2} \hat{A}_{1}^{2}(\vec{x}, t)+E_{0}(\vec{x})+\hat{F}_{0}, \\
\partial_{t} \hat{A}_{1}(\vec{x}, t)=-\left[\gamma_{1}\left(1+i \Delta_{1}\right)-i a_{1} \nabla^{2}-v \partial_{y}\right] \hat{A}_{1}(\vec{x}, t) \\
+g \hat{A}_{0}(\vec{x}, t) \hat{A}_{1}^{\dagger}(\vec{x}, t)+\hat{F}_{1} .
\end{gathered}
$$

Note that these are very similar in form to the semiclassical Eqs. (5) and (6). The Langevin operators $\hat{F}_{i}$ describe the quantum noise added as a consequence of the interaction with the bath of external modes. These have the nonvanishing second moments

$$
\left\langle\dot{F}_{1}(\vec{x}, t) \hat{F}_{j}^{\dagger}\left(\vec{x}^{\prime}, t^{\prime}\right)\right\rangle=2 \gamma_{i} \delta_{i j} \delta\left(\vec{x}-\vec{x}^{\prime}\right) \delta\left(t-t^{\prime}\right) .
$$

A direct solution of these nonlinear Langevin equations of operators is impractical, requiring the solution of an infinite hierarchy of equations for the evolution of all the products of operators that are coupled by the dynamics. A standard alternative approach to this Heisenberg picture is to consider the evolution equation of the reduced density operator $\hat{\rho}$ of the system in the Schrödinger picture and to use quasiprobability functionals. In this approach to the quantum dynamics of open problems, the intracavity dynamics is described by a master equation $[20]$ 


$$
\frac{\partial \widetilde{\rho}}{\partial t}=\frac{1}{i \hbar}[\hat{H}, \dot{\rho}]+\Lambda \hat{\rho},
$$

where $\hat{H}$ is the Hamiltonian

$$
\begin{aligned}
\hat{H}= & \hbar \int d^{2} \vec{x} \sum_{j=0,1}\left[\gamma_{j} \hat{A}_{j}^{\dagger}(\vec{x})\left(\Delta_{j}-a_{j} \nabla^{2}\right) \hat{A}_{j}(\vec{x})\right] \\
& +i v \hat{A}_{1}^{\dagger}(\vec{x}) \partial_{y} \hat{A}_{1}(\vec{x})+i E_{0}(\vec{x})\left(\hat{A}_{0}^{\dagger}(\vec{x})-\hat{A}_{0}(\vec{x})\right) \\
& +i \frac{g}{2}\left(\hat{A}_{0}(\vec{x}) \hat{A}_{1}^{\dagger}(\vec{x})-\text { H.c. }\right) .
\end{aligned}
$$

The Liouvillian $\Lambda$ accounts for dissipation through the partially reflecting mirrors of the cavity and is given by [6]

$$
\Lambda \hat{\rho}=\sum_{j=0,1} \int d^{2} \vec{x} \gamma_{j}\left\{\left[\hat{A}_{j}(\vec{x}), \hat{\rho} \hat{A}_{j}^{\dagger}(\vec{x})\right]+\left[\hat{A}_{j}(\vec{x}) \vec{\rho}, \hat{A}_{j}^{\dagger}(\vec{x})\right]\right\} .
$$

The master equation (15) can be mapped onto an equation of motion for one of a number of quasiprobability distributions in the phase space of the system [20-22]. These distributions are functionals of the $c$-number fields $\alpha_{i}(\vec{x})$ associated with the operators $\hat{A}_{i}(\vec{x})$. This evolution equation is obtained by substituting products of field operators and the density operator, depending on the ordering, by suitable operations on the distribution functionals $[6,8]$.

The evolution equations obtained in this way for the distributions are functional partial-differential equations. These are not in general of the Fokker-Planck type and do not lead to well-behaved stochastic representations in terms of Langevin equations driven by Gaussian white noise. In particular the Hamiltonian term describing the $\chi^{2}$ interaction gives a contribution

$$
\begin{aligned}
& {\left[\hat{A}_{0}(\vec{x}) \hat{A}_{1}^{\dagger}(\vec{x})-\text { H.c. }, \hat{\rho}\right]} \\
& \Leftrightarrow\left(s \alpha_{0} \frac{\delta^{2}}{\delta \alpha_{1}^{2}}+\frac{1-s^{2}}{4} \frac{\delta^{3}}{\delta \alpha_{1}^{2} \delta \alpha_{0}^{*}}\right. \\
& \left.\quad+\frac{\delta}{\delta \alpha_{0}} \alpha_{1}^{2}-2 \alpha_{0} \alpha_{1}^{*} \frac{\delta}{\delta \alpha_{1}^{\prime}}+\text { c.c. }\right) W_{s},
\end{aligned}
$$

where the parameter $s$ depends on the ordering. This term does not fulfill the requirements that guarantee a positive definite solution for $W_{s}$ : in the Wigner representation ( $s$ $=0$ ) we find third-order derivatives, while it is known [23] that positiveness requires a Fokker-Planck form of the master equation (only first- and second-order derivatives) or to include derivatives to all orders. For the $P(s=1)$ and $Q(s=$ $-1)$ representation third-order derivatives disappear, but the diffusion matrix is not positive definite so that positive solutions are again not guaranteed, although the $Q$ retains positivity through having a minimum allowed width [21]. Generally these problems have been avoided by using linearization schemes [22]. Such linearization approximations, however, are valid only for small damped fluctuations. They cannot be used in a convective regime as the reference state is unstable and the fluctuations, far from being small, are amplified. The alternative of the $P$ positive representation [24] is not suitable for the same reason and the unstable reference state results in diverging trajectories.

These problems of the convective regime can be illustrated by a comparison with the situation of a DOPO below the threshold of signal generation. In this case the stable solution is a homogeneous pump with an amplitude that depends on the coherent driving field. The signal field is zero on average, but its fluctuations show a level of selforganization that increases near the threshold. This is the regime of quantum images [4-6], noisy precursors generated by quantum noise. These images reflect the presence of eigenmodes of the linearized equations, whose eigenvalues are such that their negative real part approaches zero at threshold. The fluctuations of these eigenmodes are the least damped ones and dominate the dynamics of the signal. The important point is that the intensity of such quantum images of the signal is of the order of the quantum noise, while the pump has a macroscopic mean value. It is then possible to neglect the fluctuations in the pump, approximating it by a classical coherent field [22]. In this approximation the Hamiltonian is a quadratic function of the quantum operators. The consequence is that a well-defined Fokker-Planck equation for the Wigner distribution is obtained. Such Fokker-Planck equations can be represented in terms of stochastic Langevin equations for the $c$-number field $\alpha_{1}(\vec{x})$ [6]. The same type of approximation, linearizing around a pattern solution [8], is generally possible in the absolutely unstable regime above the threshold. A common feature of these two regimes (absolutely stable and unstable) is that the quantum noise does not change drastically the solution with respect to the stable deterministic solution. This means that in the stochastic representation, fluctuations only induce the trajectory to visit a small region in the phase space in the neighborhood of the deterministic solution. In the convective regime the classical deterministic solution is unstable and macroscopically different from the stochastic solution. In this regime the quantum noise in the DOPO is amplified, destroying the zero-valued homogeneous deterministic solution for the down-converted field and driving the system into noisesustained states having a macroscopic number of photons.

\section{TIME-DEPENDENT PARAMETRIC APPROXIMATION}

In this section we propose an approximate description of the quantum dynamics of the DOPO in the convective regime, based on the main physical features of this regime. Our aim is to be able to treat the macroscopic quantum fluctuations associated with the signal field in the convective regime.

In the convective regime there are large signal fluctuations around the unstable solution $A_{1}=0$. The coupling of the signal and the pump gives the nonlinear saturation for these amplified fluctuations. On the other hand, the pump field is always macroscopic and stable, with small damped fluctuations. This suggests the approximation of neglecting the quantum noise in the pump and approximating it by a classical field $\mathcal{A}_{0}(\vec{x}, t)$. In this way we obtain a Hamiltonian that is quadratic in the operators describing the quantum dynamics of the signal field. For such quadratic Hamiltonians, the 
Wigner quasiprobability functional of the complex function $\alpha_{1}(\vec{x}, t)$ obeys the following Fokker-Planck equation in which the classical pump field $\mathcal{A}_{0}(\vec{x}, t)$ appears parametrically

$$
\begin{aligned}
& \frac{\partial W\left(\alpha_{1} ; \mathcal{A}_{0}\right)}{\partial t} \\
& =\left[-\left(\frac{\delta}{\delta \alpha_{1}} \gamma_{1}\left[\left(1+i \Delta_{1}\right)-i a_{1} \nabla^{2}-v \partial_{y}\right] \alpha_{1}(\vec{x}, t)\right.\right. \\
& \left.\left.\quad+g \mathcal{A}_{0}(\vec{x}, t) \alpha_{1}^{*}(\vec{x}, t)+\text { c.c. }\right)+\gamma_{1} \frac{\delta^{2}}{\delta \alpha_{1} \delta \alpha_{1}^{*}}\right] W\left(\alpha_{1} ; \mathcal{A}_{0}\right) .
\end{aligned}
$$

The associated Langevin equation that represents the stochastic dynamics of the signal field $\alpha_{1}(\vec{x}, t)$ is

$$
\begin{aligned}
\partial_{t} \alpha_{1}(\vec{x}, t)= & -\gamma_{1}\left[\left(1+i \Delta_{1}\right)-i \alpha_{1} \nabla^{2}-v \partial_{y}\right] \alpha_{1}(\vec{x}, t) \\
& +g \mathcal{A}_{0}(\vec{x}, t) \alpha_{1}^{*}(\vec{x}, t)+\sqrt{\gamma_{1}} \xi_{1}(\vec{x}, t),
\end{aligned}
$$

where $\xi_{1}(\vec{x}, t)$ is a complex Gaussian white noise [see Eq. (3)]. This noise term accurately represents the effects of vacuum fluctuations associated with cavity losses on the signal field. We note that treating the pump field classically in this way is a natural extension of the parametric approximation to three-mode interactions, which treats a strong mode classically and has been widely used in quantum optics for many years [25].

It is important to note that $\mathcal{A}_{0}(\vec{x}, t)$ cannot be replaced with an expectation value of $\left\langle\hat{A}_{0}\right\rangle$ as would be possible in the regime of absolute stability (quantum images). Such an ansatz decorrelates the pump modes from the subharmonic ones and eliminates the saturation effect of the pump. In fact, with such an ansatz Eq. (16) becomes linear, giving a Gaussian probability distribution for the signal modes. This distribution would always be centered on zero, but with statistical moments that diverge above the threshold because the signal modes are undamped in the convective regime. Therefore, the stochastic differential equation must be solved selfconsistently with an equation defining the dynamics of the classical field $\mathcal{A}_{0}$. The equation we propose for $\mathcal{A}_{0}$ is suggested by the Heisenberg equation (12), with $\hat{A}_{0}$ replaced with a classical field $\mathcal{A}_{0}$. We first neglect the noise source in Eq. (12) since quantum fluctuations entering the cavity are unimportant, as compared with the macroscopic fluctuations of the signal term $\hat{A}_{1}^{2}$. Second, we replace the operator $\hat{A}_{1}^{2}$ by the $c$-number function $\alpha_{1}^{2}$ associated with our stochastic representation of the signal. This replacement is independent of operator ordering and hence will be the same should we use a different quasiprobability. This procedure gives a partialdifferential equation for the "classical" pump field driven by the $c$-number representation of the quantum signal field

$$
\begin{aligned}
\partial_{t} \mathcal{A}_{0}(\vec{x}, t)= & -\gamma_{0}\left[\left(1+i \Delta_{0}\right)-i a_{0} \nabla^{2}\right] \mathcal{A}_{0}(\vec{x}, t) \\
& -\frac{y}{2} \alpha_{1}^{2}(\vec{x}, t)+E_{0}(\vec{x})
\end{aligned}
$$

A justification for this equation is that its mean value coincides with the expectation value for $\hat{A}_{0}$ obtained from the operator equation (12). This procedure is reminiscent of the time-dependent refinement of the parametric approximation described in some detail by Kumar and Mehta [26]. This approach allows for the quantum evolution of the weak fields to feed back and affect the classical strong field. In the approach of Kumar and Mehta, this feedback is via quantum expectation values of operators for the weak fields. Here, however, we are required to take explicit account of the noisy properties of the quantum subharmonic field. We do this by using the $c$-number representation of the quantum field, associated with our stochastic simulation of it, as a term in Eq. (17).

In summary, our time-dependent parametric approximation is defined by stochastic classical equations in the Wigner representation for the fields $\mathcal{A}_{0}$ and $\alpha_{1}$, which, with the scaling (4), are

$\partial_{t} \mathcal{A}_{0}(\vec{x}, t)=-\left[\left(1+i \Delta_{0}\right)-i \nabla^{2}\right] \mathcal{A}_{0}(\vec{x}, t)-\frac{1}{2} \alpha_{1}^{2}(\vec{x}, t)+E_{0}(\vec{x})$,

$$
\begin{aligned}
\partial_{t} \alpha_{1}(\vec{x}, t)= & -\left[\left(1+i \Delta_{1}\right)-2 i \nabla^{2}-v \partial_{y}\right] \alpha_{1}(\vec{x}, t) \\
& +\mathcal{A}_{0}(\vec{x}, t) \alpha_{1}^{*}(\vec{x}, t)+\frac{1}{a^{D / 4}} \frac{g}{\gamma} \xi_{1}(\vec{x}, t) .
\end{aligned}
$$

Stochastic averages of the $c$-number variable $\alpha_{1}(\vec{x}, t)$ will provide symmetrically ordered averages of the quantum fluctuations in the signal field as driven by the "classical" pump field. The classical pump field is driven by the macroscopic quantum fluctuations in the signal as represented by the $c$-number representation $\alpha_{1}(\vec{x}, t)$. This time-dependent parametric approximation appears useful in situations in which there are large fluctuations of the signal that cannot be described by approximations based on linearization.

\section{STOCHASTIC TRAJECTORIES AND WIGNER DISTRIBUTION FUNCTION}

Numerical simulation of the stochastic trajectories associated with the Langevin equations (18) and (19) gives a good intuitive understanding of the dynamical properties of the regime below the threshold, the convective regime and the absolutely unstable regime. In this section we present such numerical simulations working with a single transverse dimension $(D=1)$ [27].

Figure 2 is a space-time plot of the near field for the signal in the below-threshold, convective and absolutely unstable regimes. Figure 3 gives the far fields associated with the same simulations. In the following we discuss the different properties of these trajectories and how they are reflected in the associated Wigner distribution. In particular, we consider the phase-space dynamics of the most intense modes of the signal pattern. The Wigner probability distribution associated with these modes displays distinctly non-Gaussian features in the convective regime. These are a result of the interplay of nonlinear and walk-off effects. It is clear that they cannot be described within a linearization scheme that 

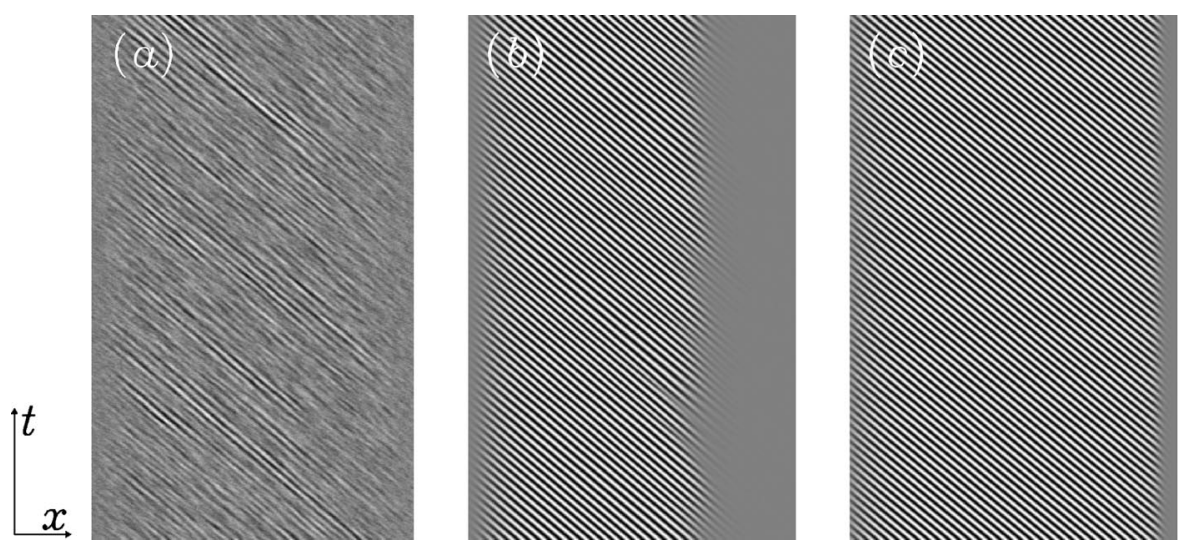

FIG. 2. Evolution of the near field of the real part of the signal $\operatorname{Re}\left[\alpha_{1}(x, t)\right]$ for (a) $F=0.999$, (b) $F=1.025$, and (c) $F=1.1$, in 2500 time units. Parameters are $\Delta_{0}=0, \Delta_{1}=-0.25, v=0.42$, system size $=1.7678 \times 512$ pixels $\simeq 900$ space units. Only the regions in which the signal is excited are shown, that is, the region of the plateau of the super-Gaussian pump [28].

does not take account of this interplay between the signal and pump fields.

\section{A. Below threshold}

Below but close to the threshold we find weakly damped fluctuations that are a precursor to the traveling pattern that appears at the threshold. The fluctuations impose a degree of spatial self-organization in those regions in which the pump is sufficiently strong to bring the OPO close to the threshold. In Fig. 2(a) we plot the real part of the stochastic variable $\alpha_{1}(x, t)$ for a single trajectory. This is a realization of these fluctuations for a pump with a super-Gaussian profile [28]. Noisy patterns of this form have been predicted for the below-threshold OPO without the walk-off and have been termed quantum images [4-6]. Not too close to threshold, the damped fluctuations can be analyzed with linearization procedures [6] in the limit of small fluctuations. Our nonlinear quantum equations enable us to study also the regime closer to the threshold, where large critical fluctuations are expected to occur. Note, in particular, that the results in Fig. 2(a) were obtained for $F=0.999$.
The selection of a preferred wave number in the stochastic pattern of Fig. 2(a) becomes more evident in the far field shown in Fig. 3(a). It is clear that there are preferred values of the wave number but that a broad distribution of weakly damped modes around these preferred $k_{c}$ and $-k_{c}$ modes is apparent.

An interesting characterization of the stochastic dynamics in the far field, Fig. 3(a), is obtained by looking at the time evolution of the stochastic amplitudes for the most intense modes $\alpha_{1}\left(k_{c}, t\right)$. We first recall that the linear stability analysis of Sec. II identifies the existence of a nonvanishing frequency $[\omega(k)=v k]$ at the threshold caused by the walkoff. This implies that a traveling pattern will emerge above the threshold and that the corresponding Fourier modes will oscillate at this frequency. We can remove this time dependence by working in a frame rotating at this frequency. This corresponds to factoring out a time factor $\varepsilon^{i \omega(k) t}$ to obtain the slowly varying amplitudes $\alpha_{1}^{\prime}(k, t)=\alpha_{1}(k, t) e^{-i \omega(k) t} . \quad \mathrm{A}$ phase-space trajectory for the slowly varying amplitude of the dominating Fourier component $\alpha_{1}^{\prime}\left(k_{c}, t\right)$ is shown in Fig. 4(a).
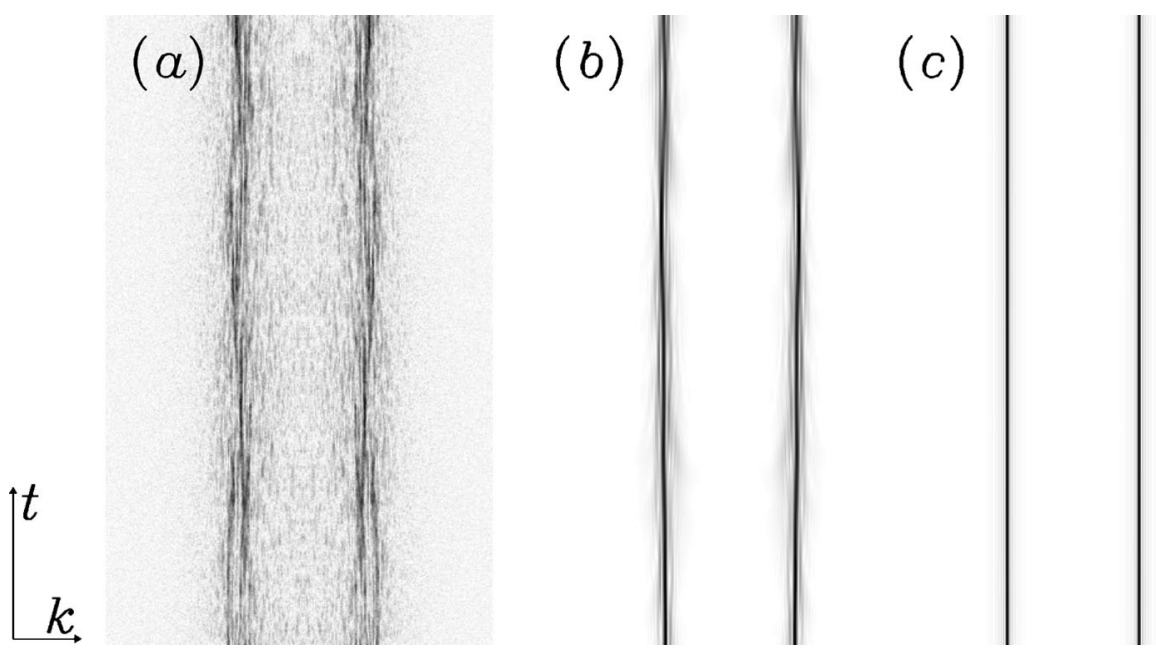

FIG. 3. Evolution of the far field $\left|\alpha_{1}(k, t)\right|$ for (a) $F=0.999$, (b) $F=1.025$, (c) $F=1.1$. Same parameters as in Fig. 2. 

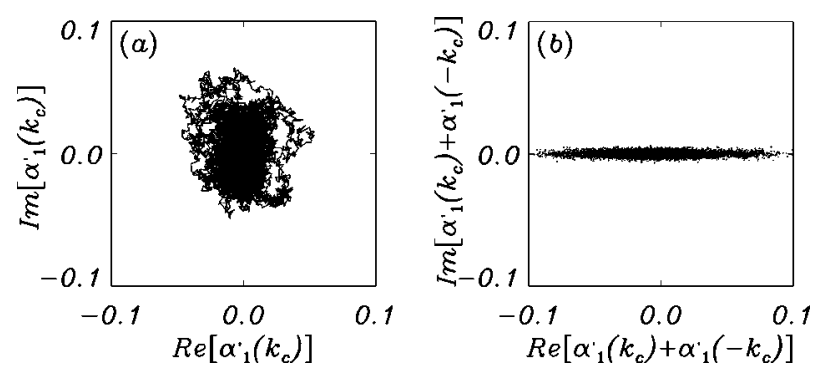

FIG. 4. (a) Trajectory of the slowly varying amplitude $\alpha_{1}^{\prime}$ $\left(+k_{c}\right)$ during 20000 time units. (b) Trajectory of $\left[\alpha_{1}^{\prime}\left(+k_{c}\right)+\alpha_{1}^{\prime}\right.$ $\left(-k_{c}\right)$ ] during 20000 time units. $F=0.999$, other parameters are as in Fig. 2, except for $d x=51 \lambda_{c} / 512 \simeq 1.7702$, where $\lambda_{c}=2 \pi / k_{c}$, 512 is the number of grid points.

The linear stability analysis of Sec. II also identified the direction of instability $V_{+}$. In particular, in the case of a real pump, and for the critical mode $k_{c}$, this direction is given by $\left[\alpha_{1}\left(+k_{c}\right)+\alpha_{1}^{*}\left(-k_{c}\right)\right]$. As a consequence the superposition of modes $\left[\alpha_{1}\left(+k_{c}\right)+\alpha_{1}\left(-k_{c}\right)\right]$ can be decomposed in two quadratures, one corresponding to the direction of instability that becomes undamped at the threshold $\left(\operatorname{Re}\left[\alpha_{1}\left(+k_{c}\right)+\alpha_{1}\right.\right.$ $\left.\left.\left(-k_{c}\right)\right]\right)$, and the orthogonal quadrature $\left(\operatorname{Im}\left[\alpha_{1}\left(+k_{c}\right)+\alpha_{1}\right.\right.$ $\left.\left.\left(-k_{c}\right)\right]\right)$ that remains damped. We observe that the superposition of slowly varying modes $\alpha_{1}^{\prime}\left( \pm k_{c}, t\right)$ can be decomposed into damped and undamped quadrature in the same way. In fact due to the symmetry $\omega(k)=-\omega(-k)$ we have $V_{ \pm}(\vec{k},-\vec{k})=e^{i \omega(k) t}\left[e^{i \Phi} \pm \delta A_{1}^{\prime}(\vec{k}) \pm \delta A_{1}^{\prime *}(-\vec{k})\right]$, so that the relative phase $e^{i \Phi_{\perp}}$ between the slowly varying modes is the same as that in the Eq. (10). Hence, we can also identify the real and imaginary quadratures of the superposition of modes $\left[\alpha_{1}^{\prime}\left(+k_{c}\right)+\alpha_{1}^{\prime}\left(-k_{c}\right)\right]$ as damped and undamped at threshold. The corresponding time trajectory of this superposition of modes displays very clearly the expected reduction of fluctuations in the damped imaginary quadrature [see Fig. 4(b)].

From the stochastic trajectories that randomly visit the different points of phase space it is easy to construct a relative histogram giving a probability density in this phase space. This density is identified with the Wigner distribution. As with all Wigner functions, the marginal distributions, obtained for one field quadrature by integrating over the orthogonal quadrature, are true probability distributions for the remaining quadrature. At a finite distance from threshold the Wigner distribution $W\left(\alpha_{1}(k)\right)$ for the field $\alpha_{1}(k)$ obtained in this way has a Gaussian shape consistent with a linearized analysis of fluctuations. Such a Gaussian Wigner distribution is a solution of the Fokker-Planck equation for the Wigner representation of linear signal fluctuations. If we consider the Wigner distribution for the superposition of modes discussed above $W\left(\alpha_{1}\left(+k_{c}\right)+\alpha_{1}\left(-k_{c}\right)\right)$, then we obtain a Gaussian centered on the origin but with a variance that depends on the orientation in the phase space [29]. There is an axis with a reduced variance (squeezed) and the orthogonal one with a larger variance (antisqueezed) (see Fig. 5). These features reflect the asymmetry or phase sensitivity of the fluctuations already visualized in the stochastic trajectory.

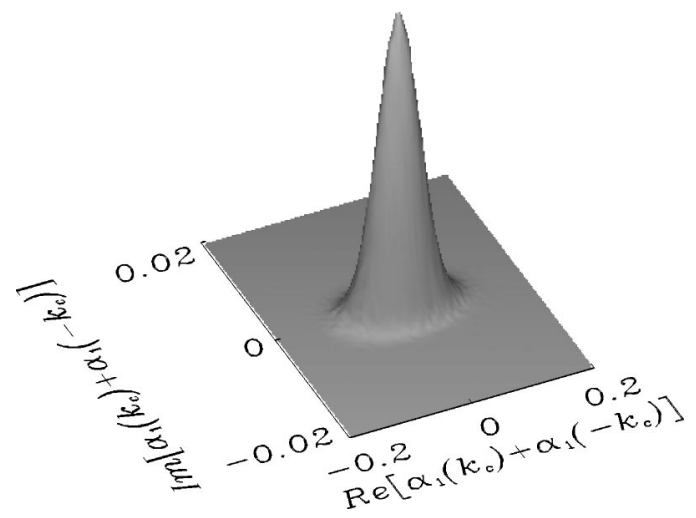

FIG. 5. Wigner distribution for the superposition of modes $\alpha_{1}\left(+k_{c}\right)+\alpha_{1}\left(-k_{c}\right)$. Parameters of Fig. 4. Total time 2000000 units. Note the factor of 10 difference in the scale of the two axes.

\section{B. Convective regime}

Differences between the regime below the threshold and the convective regime are clearly seen both in the near and far signal fields. We observe a macroscopic traveling pattern in the near field [Fig. 2(b)]. This is clearly associated with wave numbers distributed around the value of the selected one $\left(k_{M}\right)$ in the far field [Fig. 3(b)]. The spectrum of excited wave numbers is clearly narrower in the convective regime than below the threshold. This is reflected in the more regular pattern appearing in the near field. Our simulations display the typical features associated with the convective regime [13].

(1) The noise-sustained pattern does not fill the whole region in which the pump has a value above the threshold. This is because the pattern grows while traveling in the walk-off direction. Note that the space point at which the pattern reaches a macroscopic observable value changes randomly from time to time. This reflects the origin of the pattern in (quantum) noise.

(2) The far field shows the predominance of different wave numbers at different times resulting in a spatial spectrum that is broader than that found in the absence of the walk-off or in the absolutely unstable regime. There is competition between the modes within this broad spectrum and hence it is not possible to define, in this regime, a single wave number $k_{M}$ corresponding to the most excited modes. Modes with different wave numbers compete to form the pattern, switching on and off as the pattern evolves.

Phase-space trajectories for this regime are shown in Fig. 6 . We find that there are random changes in the phase and amplitude of the slowly varying signal $\alpha_{1}^{\prime}\left(+k_{c}\right)$ around a zero mean value [Fig. 6(a)]. This is similar to the behavior depicted in Fig. 4(a) below the threshold. The difference is that in the convective regime macroscopic intensities are reached, with the signal amplitude taking values comparable to those reached in the absolutely unstable regime [compare scales of Figs. 4(a), 6(a), and 9]. The continuous changes in intensity from zero to macroscopic values originate in the fact that, in the convective regime, a given mode is not constantly switched on [see Fig. 3(b)]. The pattern is sustained by the noise and is subject to a continuous renovation: different stripe patterns (with different wave numbers) grow, 

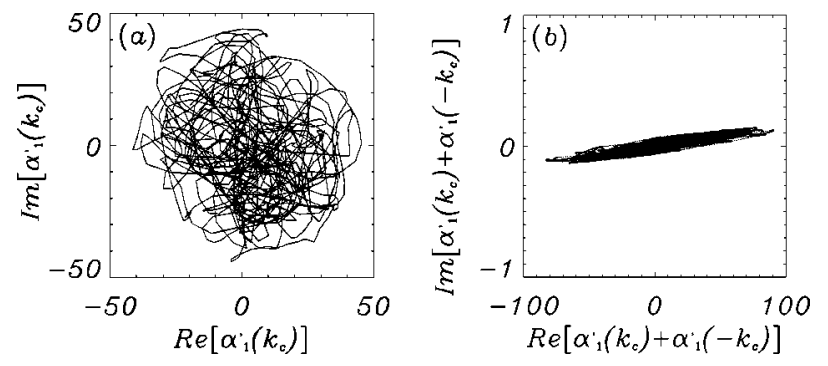

FIG. 6. (a) Trajectory of slowly varying amplitude of $\alpha_{1}^{\prime}$ $\left(+k_{c}\right)$ during 100000 time units. (b) Trajectory of $\left[\alpha_{1}^{\prime}\left(+k_{c}\right)\right.$ $\left.+\alpha_{1}^{\prime}\left(-k_{c}\right)\right]$. Parameters $F=1.025, \Delta_{0}=0, \Delta_{1}=-0.25, v=0.42$, and $d x \simeq 1.7702$.

travel in the system starting from the noise and die out. This has an important consequence in the time scales of the far field dynamics: below the threshold these scales are determined by noise, while in the convective regime they are determined by the time needed for a perturbation to travel through the system. Another indication of the nonlinear dynamics of fluctuations that occur in this regime is that the quadrature displaying reduced fluctuations is no longer the one determined by the linear analysis. This is seen in Fig. 6(b) where the ellipse of fluctuations is tilted with respect to the corresponding one below threshold Fig. 4(b).

The probability distributions obtained from the trajectories of Fig. 6 also reflect the nonlinear nature of the fluctuations in this regime. In Fig. 7 we show the $W$ distribution for the superposition of modes $\left[\alpha_{1}(+k)+\alpha_{1}(-k)\right]$ for one of the most excited wave numbers, namely $k=1.04 k_{c}$. A most noticeable feature is the non-Gaussian shape of the distribution for large values of the amplitude in the direction of undamped fluctuations. The wings of the distribution originate in the macroscopic fluctuations of the mode under consideration when it switches on. Its most probable value is still zero, reflecting the fact that most of the time the mode remains switched off. We can view these non-Gaussian features in the wings of our Wigner functions as precursors of the pair of peaks appearing in the absolutely unstable regime. These wings become more pronounced as we approach the absolutely unstable regime.

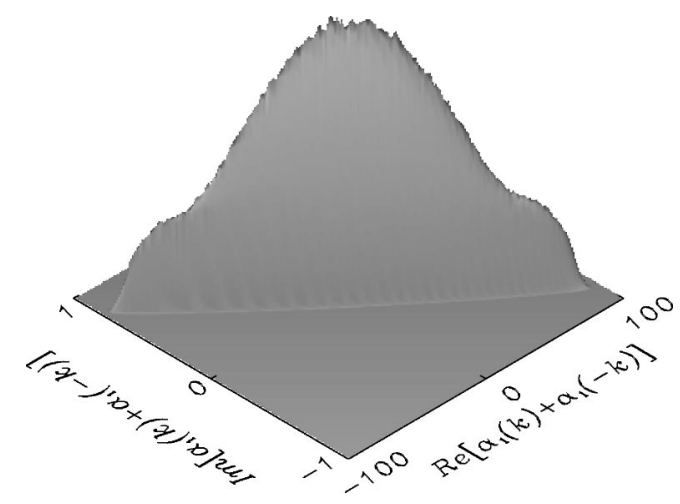

FIG. 7. $W\left(\alpha_{1}(+k)+\alpha_{1}(-k)\right)$, for an excited mode $k$ $=1.04 k_{c}$, obtained from a trajectory during 10000000 time units. Other parameters as in Fig. 6.

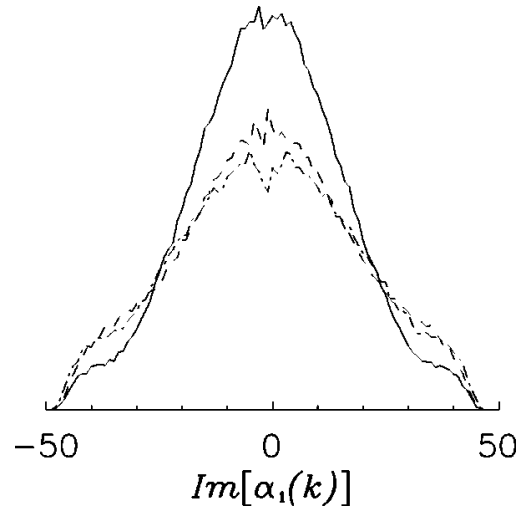

FIG. 8. Section of the Wigner distribution along the imaginary axis $W\left(0, \operatorname{Im}\left[\alpha_{1}(k)\right]\right)$ for three excited modes: $k_{c}$ (dashed line), $k^{\prime}$ $=1.04 k_{c}$ (dashed dot line), and $k^{\prime \prime}=1.06 k_{c}$ (continuous line). Same parameters as Fig. 6.

Finally, we note that the modes that become excited and contribute to the dynamics seem to reach a common maximum amplitude. This is probably fixed by the maximum value of the energy exchanged with the pump mode in the nonlinear interaction. This is shown in Fig. 8 where the possible values of different modes are seen to be cut off at essentially the same amplitude. The non-Gaussian form of these distributions is also clear and this again demonstrates that we are dealing with nonlinear effects associated with the quantum fluctuations.

\section{Absolutely unstable regime}

In the absolutely unstable regime we observe from the near field plot, Fig. 2(c), that a macroscopic and stable traveling pattern fills the whole of the above-threshold region. This behavior is reflected in the far field, Fig. 3(c), which shows a well-defined and fixed dominant wave number and a narrow spatial spectrum. We should note that the dominant wave number $k_{M}$ does not coincide with the most unstable wave number at the threshold $k_{c}$. This is a consequence of the interplay between nonlinearities and the walk-off. Phasespace trajectories for the amplitudes of these two modes are shown in Fig. 9. Even after elimination of the rapid frequency there remains a phase-diffusion process, but macroscopic values of the intensity are maintained. Although there is essentially only the phase diffusion for $k_{M}$, the critical mode, with wave number $k_{c}$, displays a second frequency superimposed on the phase-diffusion process.
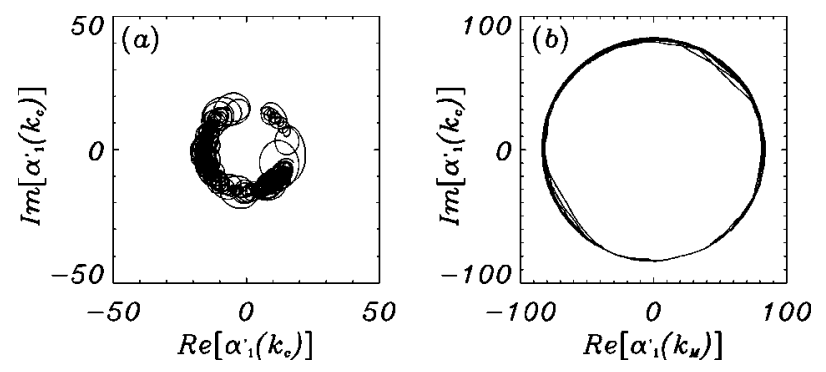

FIG. 9. Trajectories of (a) $\alpha_{1}^{\prime}\left(k_{c}\right)$ and (b) $\alpha_{1}^{\prime}\left(k_{M}\right)$ during 100000 time units, $F=1.05$, other parameters as in Fig. 6 . 


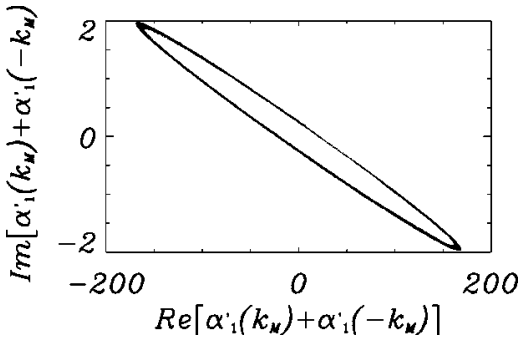

FIG. 10. Trajectory of $\left[\alpha_{1}^{\prime}\left(+k_{M}\right)+\alpha_{1}^{\prime}\left(-k_{M}\right)\right]$ over 10000000 time units. Other parameters as in Fig. 9.

The phase-space trajectory for the superposition of modes $\left[\alpha_{1}^{\prime}\left(\vec{k}_{M}, t\right)+\alpha_{1}^{\prime}\left(-\vec{k}_{M}, t\right)\right]$ is shown in Fig. 10. We observe that fluctuations are not uniformly distributed around a zero value as they were in the below threshold (Fig. 4) and convective (Fig. 6) regimes. Instead, they describe a closed curve around the origin. The associated $W$ distributions display peaks at two values. These correspond to the two points of the maximum curvature of the elliptical ring.

The main characteristics of the trajectories in the phase space are reflected in the associated Wigner distributions. For the less-intense modes contributing to the dynamics we can approximate the associated Wigner function $W\left(\alpha_{1}(k)\right)$ by a Gaussian, displaced from and orbiting about the origin in the phase space. In Fig. 11(a) we show a cut along the real direction of the Wigner distribution for the critical mode $\left(W\left(\operatorname{Re}\left[\alpha_{1}\left(k_{c}\right)\right], 0\right)\right)$. By contrast, the most-intense mode (with wave number $k_{M}$ ) displays some interesting new features. Figure 11(b) shows an asymmetry in the distribution of fluctuations around the mean amplitude in each of the peaks, with a sharp decay of the distribution at some maximum amplitude. These facts indicate the existence of nonlinear properties associated with the quantum fluctuations in the absolutely unstable regime. These features would necessarily be absent in any analysis based on a linearization about a deterministic macroscopic state.

\section{NONCLASSICAL PROPERTIES IN THE CONVECTIVE REGIME}

The convective regime is characterized by amplified fluctuations and macroscopic noisy patterns. It is interesting to
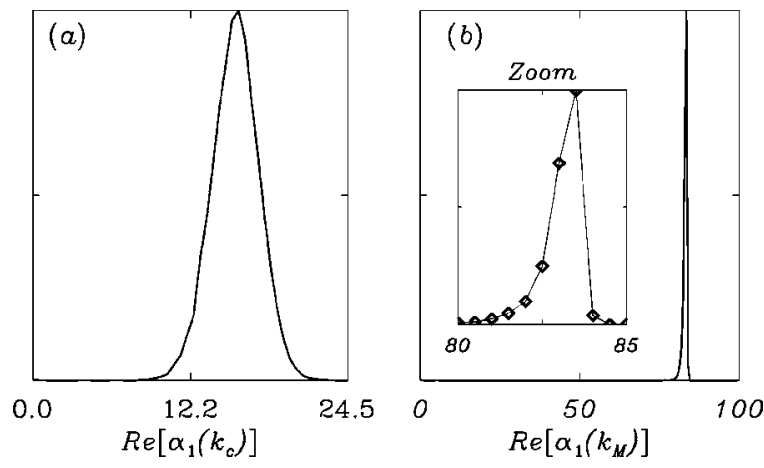

FIG. 11. $W\left(\operatorname{Re}\left[\alpha_{1}(k)\right], 0\right)$, for positive values of $\operatorname{Re}\left[\alpha_{1}(k)\right]$, for (a) $k=k_{c}$, (b) $k=k_{M}$, obtained from a trajectory during 10000000 time units. Other parameters as in Fig. 9. These figures are symmetric around zero.

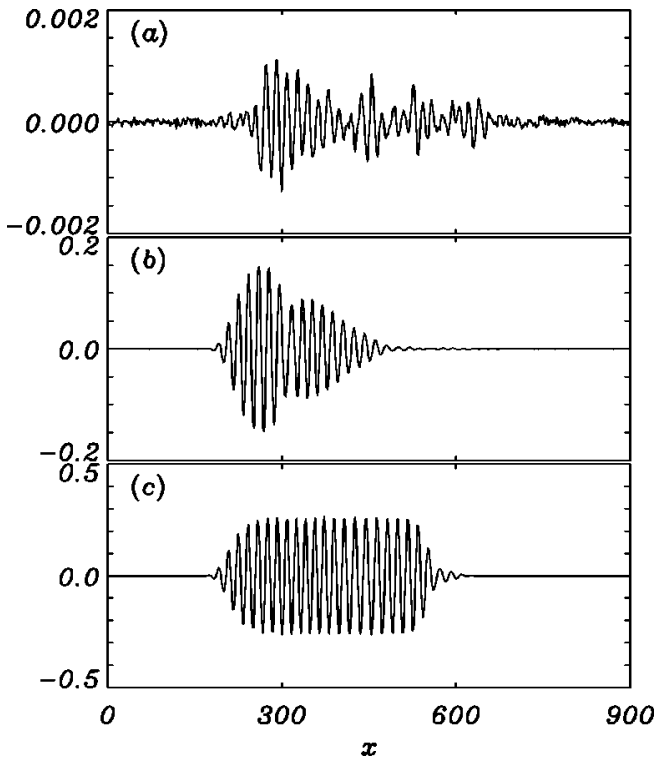

FIG. 12. Snapshots of the real part signal $\operatorname{Re}\left[\alpha_{1}(x)\right]$ for different pump values: (a) $F=1.001$, (b) $F=1.01$, and (c) $F=1.025$. Other parameters are $d x=1.7678,512$ grid points, $a_{0}=1, \Delta_{0}=0, \Delta_{1}$ $=-0.25$, and $v=0.42$. Note the different vertical scales in the figures.

ask, therefore, if any of the low-noise quantum features found below the threshold can survive in this noisy environment. Quantum effects in the OPO have been observed as subshot noise fluctuations both in the field quadratures and intensity differences associated with the down-converted light [30]. Examples of the noisy features associated with the real part of the signal field in this regime are plotted in Fig. 12 for three different values of the driving field, all within the convective regime. Note the different scales on the vertical axes in these figures.

It is helpful, in looking for nonclassical effects, to keep in mind the manner in which such effects appear below the threshold. We will also restrict ourselves to the study of quantum correlations in the far field. Conditions for squeezing and associated nonclassical effects are usually expressed in terms of normally ordered moments of operators (indicated by ::). These can be obtained from the symmetrically ordered moments [indicated by $S()$ ], that are associated with the Wigner function, by use of the commutation relations (11)

$$
\begin{gathered}
\left\langle: \hat{A}(k, t) \hat{A}\left(k^{\prime}, t\right):\right\rangle=\left\langle S\left(\hat{A}(k, t) \hat{A}\left(k^{\prime}, t\right)\right)\right\rangle, \\
\left\langle: \hat{A}(k, t) \hat{A}^{\dagger}\left(k^{\prime}, t\right):\right\rangle=\left\langle S\left(\hat{A}(k, t) \hat{A}^{\dagger}\left(k^{\prime}, t\right)\right)\right\rangle-\frac{1}{2} \delta\left(k-k^{\prime}\right) .
\end{gathered}
$$

The $\delta$ function appearing in the second of these equations is a signature of the shot or vacuum noise. Our approximation scheme is based in the Wigner representation and gives results for correlations of symmetrically ordered operators for the intracavity fields. In order to obtain results for the corresponding normally ordered products and to test for the presence of nonclassical effects, we need to establish a reference shot noise level. This level can be obtained for each quadra- 


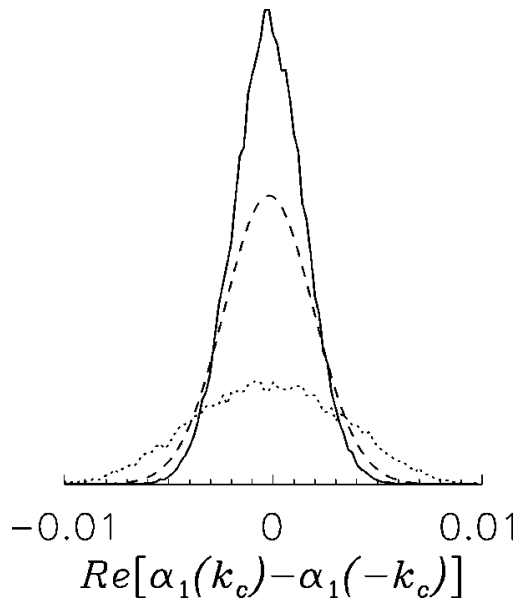

FIG. 13. $W\left(\operatorname{Re}\left[\alpha_{1}\left(k_{c}, t\right)-\alpha_{1}\left(-k_{c}, t\right)\right], 0\right)$. Continuous line is obtained for $F=1.001$, and dotted line for $F=1.01$. The dashed line represents the distribution for the vacuum state, corresponding to the shot noise level. The distributions are relative to trajectories of 2000000 time units. Other parameters as in Fig. 12.

ture correlation from the variance of the linear stochastic process associated with the empty cavity

$$
\partial_{t} s(x, t)=-\left[\left(1+i \Delta_{1}\right)-2 i \nabla^{2}\right] s(x, t)+\frac{1}{a^{1 / 4}} \frac{g}{\gamma} \xi_{1}(x, t) .
$$

Here we have omitted the walk-off term as it does not affect the shot noise level. Squeezing in our simulations will be associated with a quadrature probability distribution that is narrower than the Gaussian associated with this linear process. In general, we can consider a different quadrature for each wave number $k$. It is useful to define a pair of (superposition mode) quadratures for each $k$ parametrized by the angle $\theta$. For the critical wave number these take the form

$$
\hat{X}_{ \pm}(0)=\frac{1}{2}\left[\hat{A}_{1}\left(k_{c}, t\right) \pm \hat{A}_{1}\left(-k_{c}, t\right)\right] e^{i \theta}+\text { H.c. }
$$

We expect, in general, that the most strongly squeezed quadrature should depend on the value of $\theta$ [7].

We begin our investigation of the convective regime at a point that is just above the threshold with $F=1.001$ [Fig. 12(a)]. Fluctuations associated with the pattern are in this case still relatively small and we find that the Wigner distribution has a Gaussian shape as shown in Fig. 13. We find that there is quadrature squeezing, with the squeezed quadrature $\hat{X}_{-}(0)$ exhibiting the same level of squeezing as is found just below the threshold. In particular, for $F=F_{\mathrm{thr}}$ \pm 0.001 we find that the intracavity field is squeezed by $50 \%$ below the shot noise limit for a flat pump and by $37 \%$ for a super-Gaussian pump [31]. This indicates a smooth variation across the threshold for the squeezed quadrature variance. For excited modes, other than the critical one, we also find squeezing below the shot noise level for the appropriate quadrature.

Increasing the value of the pump, so as to move further into the convective regime, leads to a rapid increase in the magnitude of the signal field. Indeed, for $(F=1.01)$ we ob-

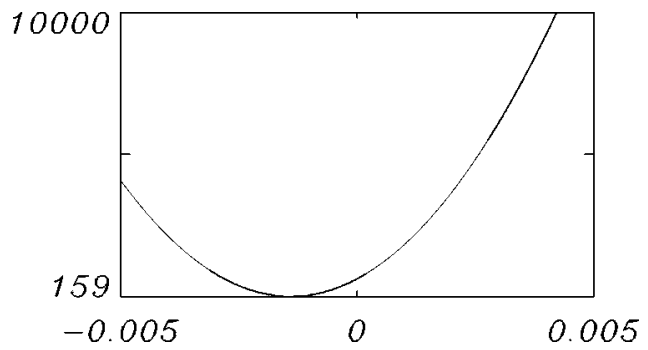

FIG. 14. $\operatorname{Var}\left[\hat{X}_{-}(\theta)\right]$ at $F=1.025$ for the critical wave number $k=k_{c}$. The minimum occurs for $\theta<0$.

serve, in Fig. 12(b) that the signal field has grown by two orders of magnitude. Fluctuations are still extremely phase sensitive and, as depicted in Fig. 6(a) there is a strong reduction in the fluctuations for some quadratures. This reduction is insufficient, however, to reach below the shot noise level and there is no squeezing. In fact, we find residual fluctuations $+27 \%$ above the shot noise level. This is comparable with the value associated with the coherent states. These enhanced fluctuations are associated with a much broader Wigner distribution as shown in Fig. 13. It is remarkable, however, that this enhanced but still small level of fluctuation can coexist with the macroscopic fluctuations in orthogonal quadrature. If we move still further above the threshold then we find, for $F=1.025$ [Fig. 12(c)], a variance which is 159 times the shot noise level and both quadratures display fluctuations that are well above the level usually associated with quantum effects. We note that for the parameter values used here, the threshold of absolute instability for an infinite system occurs at $F \sim 1.035$.

A further indication of the nonlinear nature of the fluctuations in the convective regime is given by the fact that the angle $\theta$, for which there is the greatest reduction in the fluctuations, changes with the strength of the pump value. This has already been discussed in connection with Fig. 6(a). In particular, for the critical wave number, $\hat{X}_{-}(\theta)$ shows strongest squeezing for $\theta=0$ in the linear regime below the threshold. In the convective regime, however, the greatest reduction in the quadrature fluctuations occurs for a value of $\theta<0$. This is shown in Fig. 14 in which we plot the variance $\operatorname{Var}\left[\hat{X}_{-}(\theta)\right]$ in normal ordering and normalized to the shot noise level, for $F=1.025$.

The OPO can also exhibit strong correlations between the far field intensities associated with opposite wave numbers. We have calculated the fluctuations in the intensity difference for opposite wave numbers associated with the normally ordered moment $\left\langle:\left[\hat{A}_{1}^{\dagger}(k) \hat{A}_{1}(k)-\hat{A}_{1}^{\dagger}(-k) \hat{A}_{1}\right.\right.$ $\left.(-k)]^{2}:\right\rangle$. A negative value for this quantity indicates a nonclassical effect, sometimes referred to as twin beams or intensity difference squeezing [32]. As in our discussion of quadrature squeezing, we find that this quantity is only negative very near to the threshold $(F=1.001)$. Further into the convective regime we find that the macroscopic noise associated with the formation of a pattern increases the noise in the intensity difference. For $F=1.01$ we find that the intensity-difference squeezing has been replaced with fluctuations in excess of the shot noise level. 
In summary, we have shown that quantum effects can survive above the threshold in the convective regime but only very near to the threshold. On increasing the pump and entering further into the convective regime, we find that nonlinear effects associated with the fluctuations tend to distribute part of the macroscopic fluctuations into the observables that are squeezed nearer to the threshold. This identifies the walk-off as an effective mechanism of quantum decoherence in which the macroscopic nonlinear fluctuations present in the convective regime overwhelm quantum effects associated with noise reduction.

\section{CONCLUSION}

We have introduced a suitable method to describe the quantum properties of macroscopic patterns sustained by quantum fluctuations in a degenerate optical parametric oscillator with walk-off. These patterns appear in the convective regime and are characterized by a broad far field spectrum with continuous competition between several wave numbers (thus, few-mode approximations are not adequate) and by being the result of amplified quantum fluctuations around an unstable reference state. Traditional linearization techniques cannot be applied in these situations. Instead we use a time-dependent parametric approximation in which the pump field is treated as a $c$-number variable but driven by the $c$-number representation of the quantum subharmonic signal field. The key point is that this includes the effects of the fluctuations in the signal on the pump, which in turn act back on the signal.

Using this method we have described the quantum fluctuations in type-I OPO with walk-off in three regimes: below the threshold of instability, in the convective unstable regime and in the absolute unstable regime.

Below the threshold we find that the Wigner representation has a Gaussian shape centered at the origin. This is the result previously found in an OPO without walk-off [6] from a linearized analysis. We also find that the walk-off does not destroy the existence of squeezing in suitable quadratures.

In the convective regime the macroscopic character of the fluctuations is reflected in an extremely broad Wigner distri- bution where the probability is still centered at the origin but the nonlinear effects lead to the appearance of the wings in the distribution that is no longer a Gaussian. These wings are, in fact, precursors of the pair of peaks appearing in the absolutely convective regime. We show that squeezing in the appropriate observables can be also obtained in this regime but only just above the threshold. The walk-off and the nonlinearities act as quantum decoherence mechanism, distributing part of the macroscopic fluctuations into the observables that were squeezed below the threshold. Another nonlinear effect appears in the selection of the quadrature displaying reduced fluctuations, that is no longer the one determined linearly.

In the absolutely unstable regime there are also clear indications of nonlinear properties associated with quantum fluctuations. The interplay between the walk-off and nonlinearity results in a complex dynamics in which the frequencies of far field modes are not constant, giving a complicated variation of the phases. Also, the most-intense mode is not the critical mode. We find that while the Wigner distribution for the less-intense modes can be approximated by a Gaussian (displaced from the origin and orbiting about it) this is not the case for the most-intense modes for which the distribution of fluctuations is asymmetric around the mean amplitude with a sharp decay at some maximum amplitude.

Finally, our method can be used in other situations and systems in which there are large fluctuations of the signal that cannot be described by approximations based on linearization. This includes situations in which the critical fluctuations appear at the threshold for pattern generation.

\section{ACKNOWLEDGMENTS}

This work was supported by the European Commission Projects QSTRUCT (Project No. ERB FMRX-CT96-0077) and QUANTIM (Project No. IST-2000-26019). R.Z., M.S.M., and P.C. acknowledge financial support from MCyT (Spain) Projects Nos. PB97-0141-C02-02 and BFM 20011108. S.M.B. thanks the Royal Society of Edinburgh and the Scottish Executive Education and Lifelong Learning Department for financial support.
[1] S. Ducci, N. Tréps, A. Maitre, and C. Fabre, Phys. Rev. A 64, 023803 (2001), and references therein.

[2] L. A. Lugiato and F. Castelli, Phys. Rev. Lett. 68, 3284 (1992).

[3] L. A. Lugiato, A. Gatti, and H. Wiedemann, in Quantum Fluctuations, edited by S. Reynaud, E. Giacobino, and J. Zinn Justin (Elsevier, Amsterdam, 1997).

[4] A. Gatti and L. A. Lugiato, Phys. Rev. A 52, 1675 (1995); L. A. Lugiato, A. Gatti, H. Ritsch, I. Marzoli, and G. L. Oppo, J. Mod. Opt. 44, 1899 (1997); I. Marzoli, A. Gatti, and L. A. Lugiato, Phys. Rev. Lett. 78, 2092 (1997).

[5] For a review, see for example, (a) L. A. Lugiato, Chaos, Solitons Fractals 4, 1251 (1994), and references quoted therein; (b) L. A. Lugiato, M. Brambilla, and A. Gatti, Adv. At., Mol., Opt. Phys. 40, 229 (1998).
[6] A. Gatti, H. Wiedermann, L. A. Lugiaro, I. Marzoli, G. L. Oppo, and S. M. Barnett, Phys. Rev. A 56, 877 (1997).

[7] J. Jeffers and G-L. Oppo, Phys. Rev. A 60, 2393 (1999).

[8] R. Zambrini, M. Hoyuelos, A. Gatti, P. Colet, L. Lugiato, and M. San Miguel, Phys. Rev. A 62, 63801 (2000).

[9] M. Santagiustina, P. Colet, M. San Miguel, and D. Walgraef, Phys. Rev. Lett. 79, 3633 (1997).

[10] M. Santagiustina, P. Colet, M. San Miguel, and D. Walgraef, Opt. Lett. 23, 1167 (1998).

[11] R. J. Deissler, J. Stat. Phys. 40, 371 (1985); 54, 1459 (1989); see also first work about convective systems in plasma physics: R. J. Briggs, Electron-Stream Interaction with Plasmas (MIT, Cambridge, MA, 1964).

[12] M. Santagiustina, P. Colet, M. San Miguel, and D. Walgraef, J. Opt. B: Quantum Semiclassical Opt., 1, 191 (1999). 
[13] M. Santagiustina, P. Colet, M. San Miguel, and D. Walgraef, Phys. Rev. E 58, 3843 (1998).

[14] G. Izus, M. Santagiustina, M. San Miguel, and P. Colet, J. Opt. Soc. Am. B 16, 1592 (1999).

[15] B. E. A. Saleh and M. C. Teich, Fundamentals of Photonics (Wiley, New York, 1991).

[16] G-L. Oppo, M. Brambilla, and L. A. Lugiato, Phys. Rev. A 49, 2028 (1994).

[17] H. Ward, M. N. Ouarazi, M. Taki, and P. Glorieux, Eur. Phys. J. D 3, 275 (1998).

[18] M. Taki, M. San Miguel, and M. Santagiustina, Phys. Rev. E 61, 2133 (2000).

[19] This spectrum is sharply narrowed on passing into the absolutely unstable regime [13].

[20] S. M. Barnett and P. Radmore, Methods in Theoretical Quantum Optics (Oxford Science, Oxford, 1997).

[21] C. W. Gardiner and P. Zoller, Quantum Noise, 2nd ed. (Springer, Berlin, 2000).

[22] H. Carmichael, An Open Systems Approach to Quantum Optics, Lecture Notes in Physics, Vol. m18 (Springer, Berlin, 1993).

[23] R. F. Pawula, Phys. Rev. 162, 186 (1967).

[24] A. Gilchrist, C. W. Gardiner, and P. D. Drummond, Phys. Rev. A 55, 3014 (1997).

[25] W. H. Louisell, A. Yariv, and A. E. Siegman, Phys. Rev. 124, 1646 (1961).

[26] S. Kumar and C. L. Mehta, Phys. Rev. A 21, 1573 (1980); 24,
1460 (1981), and references therein. See also S. M. Barnett and P. L. Knight, J. Opt. Soc. Am. B 2, 467 (1985).

[27] Numerical simulations are performed using a pseudospectral method as in [8]. We use the Gaussian random number generator proposed in R. Torai and A. Chakrabarti, Comput. Phys. Commun. 74, 327 (1993).

[28] The expression of super-Gaussian pump in all simulations is $E_{0}(x)=\exp \left[-1 / 2(x / 317)^{10}\right]$ with $x$ varying in the interval $[-453 ; 453]$. This produces a profile that is everywhere smooth but has a flat central plateau.

[29] In presence of a flat pump, equations for $\alpha_{1}(k, t)$ are invariant under the change $\alpha_{1}(k, t) \rightarrow e^{i \phi} \alpha_{1}(k, t)$, so that it is equivalent to construct the $W$ distribution considering trajectories $\alpha_{1}(k, t)$ or $\alpha_{1}^{\prime}(k, t)$. For nonflat pump this symmetry property is generally lost. In the pictures we represent the $W$ distribution for the amplitudes $\alpha_{1}$.

[30] L. A. Wu, H. J. Kimble, J. L. Hall, and H. Wu, Phys. Rev. Lett. 57, 2520 (1986); S. Reynaud, Europhys. Lett. 4, 427 (1987); A. Heidmann et al., Phys. Rev. Lett. 59, 2555 (1987).

[31] It is also possible to evaluate the squeezing outside the cavity. The output field arises from the interference of the intracavity field transmitted and the input field reflected by the cavity input/output mirror [M. J. Collett and C. W. Gardiner, Phys. Rev. A 30, 1386 (1984)]. The evaluation of the dynamics of the output field requires the simulation of coupled stochastic processes, as shown in [8].

[32] J. Mertz et al., Opt. Lett. 16, 1234 (1991). 